\title{
Stud. med. Brecht
}

Andreas Skrziepietz

Korrespondenz:

Andreas Skrziepietz Schierholzstrasse 94 D-30655 Hannover

askrz@web.de

www.docmacher.de
Vor 50 Jahren starb der vielleicht grösste deutschsprachige Dramatiker des 20. Jahrhunderts. In Biographien kann man lesen, dass Brecht u.a. Medizin studiert hat. Was ist dran an der Geschichte?

Wenn der Herr Stabsarzt kommandierte: «Amputieren Sie das Bein, Brecht!» antwortete ich: «Zu Befehl, Herr Stabsarzt!» und schnitt das Bein ab. Wenn man mir sagte: «Machen Sie eine Trepanation!», öffnete ich den Schädel eines Mannes und reparierte an seinem Gehirn herum. [1]

Brecht als Unfall- und Neurochirurg? Warum nicht? Dass der Dichter der Dreigroschenoper seinen Kriegsdienst als Sanitätssoldat in einem Lazarett ableistete, ist bekannt. Am 14. Januar 1918 musste er vor der Musterungskommission des 1. Armeekorps in Augsburg antreten und wurde d.g.v. F. (dauernd garnisonsverwendungsfähig Feld) geschrieben. Daran konnte auch die Überdosis Bohnenkaffee nichts ändern, die er zuvor zu sich genommen haben soll, um einen schwachen Kreislauf zu simulieren [2]. Der Stabsarzt war offenbar nicht beeindruckt. Immerhin musste Brecht nicht an die Front, sondern wurde ab 1. Oktober 1918 Sanitätssoldat, genauer gesagt: Militärkrankenwärter im Reservelazarett Augsburg.

In hohem Masse zu dieser Entscheidung beigetragen haben dürfte, neben einem Gesuch des gewiss nicht einflusslosen Vaters, immerhin Fabrikdirektor, die Tatsache, dass Brecht ab Mai 1918 Medizinstudent und als solcher, aufgrund des Mangels an medizinischem Fachpersonal, vom Dienst an der Front befreit war. Nachdem er ab dem Wintersemester 1917 an der LudwigMaximilians-Universität für Literaturwissenschaft eingeschrieben war, kam in einem Brief vom Mai 1918 an seine damalige Freundin Paula Banholzer die überraschende Mitteilung: «Ich hab mich für Medizin umschreiben lassen - eine Mords-Lauferei!» [3].

Medizin und Dichtkunst: Eine nicht gerade seltene Kombination in der deutschen Geistesgeschichte: Schiller, Büchner, Benn, Döblin und Bamm fallen einem spontan ein.

Aber bereits Wolfram v. Eschenbach hat man aufgrund seiner Beschreibung einer Pleurapunktion im «Parzival» eine medizinische Ausbildung nachgesagt [4]. Ob's stimmt, wird sich nach etwa 700 Jahren wohl nicht mehr klären lassen.
Schiller soll seine Nachtdienste im Lazarett dazu genutzt haben, die «Räuber» zu verfassen. Wenn das zutrifft, kann man sich ungefähr ausrechnen, wie viele Meisterwerke der deutschen Literatur aufgrund der seit Schillers Zeiten massiv zugenommen Arbeitsbelastung im Nachtdienst ungeschrieben blieben. Georg Büchner verdanken wir einige interessante Einblicke in die klinische Forschung im frühen 19. Jahrhundert: «Sehen Sie: der Mensch, seit einem Vierteljahr isst er nichts als Erbsen; bemerken Sie die Wirkung, fühlen Sie einmal: was ein ungleicher Puls.» [5]. Der sozialkritische Dichter des Frühexpressionismus soll angeblich zu Brechts Vorbildern gezählt haben, womit man versuchte, dessen plötzliche Entscheidung für das Medizinstudium zu begründen [6, 7]. Brecht also auf dem «Weg jener großen Schriftsteller, deren naturwissenschaftlicher Analyse wir die wesentlichen Aufschlüsse über die Wirklichkeit unseres wissenschaftlichen Zeitalters verdanken», wie Walter Jens [8] einst meinte?

Eher nicht, denn schliesslich sollte man nicht ganz vergessen, dass Brecht zu jener Zeit noch gar kein besonders scharfer Kritiker der bürgerlichen Gesellschaft und erst recht kein Marxist war - das sind Entwicklungen, die erst in der Berliner Zeit unter dem Einfluss von Karl Korsch, an dessen Abendkursen über Marxismus und Arbeiterbewegung Brecht Ende der 20er Jahre teilnahm, beginnen sollten. Vielleicht hat ein Erlebnis während einer Demonstration im Jahre 1929 in Berlin eine auslösende Bedeutung gehabt: Es hat damals, [...] über 20 Tote unter den Demonstranten gegeben. Als Brecht die Schüsse hörte und sah, wie die Menschen getroffen wurden, wurde er so weiß im Gesicht, wie ich ihn nie zuvor in meinem Leben gesehen hatte. [9]

Für den jungen Brecht jedenfalls schienen Bohemiens wie Frank Wedekind eine weitaus grössere Anziehungskraft zu besitzen, zumindest sprach er anlässlich Wedekinds Tod in einem Nachruf, der am 12. März 1918 in den «Augsburger Neuesten Nachrichten» erschien, von Wedekind als dem «großen Erzieher Europas» [10]. Und es ist wohl kein Zufall, dass sein erster Sohn Frank hiess und nicht Georg.

Eine besondere Neigung zur Naturwissenschaft lässt sich für diese Zeit bei Brecht jedenfalls auf keinen Fall nachweisen. Die Hinwen- 


\section{Literatur}

1 Esslin M. Das Paradox des politischen Dichters. Frankfurt/ Main: Athenäum; 1962

2 Mittenzwei W. Das Leben des Bertolt Brecht. Band 1. Frankfurt/Main: Suhrkamp; 1987.

3 Brecht B. Grosse kommentierte Berliner und Frankfurter Ausgabe 1993. Band 28, S. 55. Berlin/Frankfurt: Aufbau/Suhr kamp.

4 Haage B. Urjans Heilung nach der «Chirurgia» des Abu L-Quasim. ZDPh. 1985;104: 357-67.

5 Büchner G. Woyzeck. Szene: Der Hof des Doktors

6 Haas W. Bert Brecht. Köpfe des XX. Jahrhunderts, Band 7 Berlin: Colloquium; 1958.

7 Bornemann E. Ein Epitaph für Bertolt Brecht. Sinn und Form. 1957; 2. Sonderheft: 149

8 Jens W. Poesie und Doktrin. Bertolt Brecht. In: Jens W. Statt einer Literaturgeschichte. Pfullingen: Artemis und Winkler; 1962. S. 227-58.

9 Sternberg F. Der Dichter und die Ratio. Erinnerungen an Bertolt Brecht. Göttingen: Sachse und Pohl; 1963.

10 zitiert nach: Schmidt D. «Baal» und der junge Brecht. Stuttgart: Metzler; 1966.

11 Brecht, Bd. 23, S. 47.

12 Völker K. Bertolt Brecht. Eine Biographie. München: Rowohlt; 1976.

13 Brecht, Bd. 28, S. 55.

14 Hecht W. Brecht-Chronik 1898-1956. Frankfurt/Main: Suhrkamp; 1998.

15 Brecht, Bd. 1, S. 11.

16 Brecht, Bd. 1, S. $322 f$.

17 Tucholsky K. Mit 5 PS durch die Literatur. Berlin und Weimar: Aufbau; 1973.

18 Tretjakow S. Bert Brecht. International Literature (Moskau). 1937;(Heft 5):60-70. dung zum Positivismus und das Interesse für die Philosophie des «Wiener Kreises» in den späten 20er Jahren ist wohl erst im Zusammenhang der zunehmenden Sympathie für den Marxismus entstanden, der ja mit dem Positivismus zumindest das materialistische «Fundament» gemeinsam hat. Eine Aussage über die Psychoanalyse wie die folgende aus einem Brief des Jahres 1944 ist für den frühen Brecht undenkbar.

Freilich verschafft die Psychoanalyse ein anderes Vergnügen, nämlich das, möglichst viel Geld für die eigene Person auszugeben. Die Psychoanalytiker sehen bekanntlich eine starke Heilkraft im Zahlen der Patient nimmt sie ernst, weil er zahlt; sehr ernst, weil er sehr viel zahlt. [11]

Was den Wechsel zur Medizin angeht, so ist $\mathrm{zu}$ seiner Begründung vermutet worden, dass Brecht möglicherweise hoffte, durch eine medizinische Ausbildung seiner an Krebs erkrankten Mutter helfen zu können, oder dass er beabsichtigte, einst die Arztpraxis des Vaters seiner Freundin zu übernehmen. Auffällig ist die zeitliche Nähe zwischen Musterung und Fachwechsel aber auf jeden Fall. Zumal das Beispiel seines Schulfreundes Rudolf Prestel, der an der Front das linke Beine verlor, Brecht die Schrecken des Stellungskrieges plastisch vor Augen führte [2]. Hinzu kommt, dass seine medizinisch-naturwissenschaftliche Laufbahn sich offensichtlich auf den Besuch einiger Vorlesungen über deskriptive Anatomie, Infektionskrankheiten, Experimentalphysiologie, Anthropologie und auf Laborübungen in «unorganischer Chemie» beschränkt zu haben scheint, im Sektionssaal hingegen wurde er niemals gesehen [2, 12-14].

Und was ist mit den Amputationen und Trepanationen, die er angeblich durchgeführt haben soll? Es ist behauptet worden, dass ihm in diesem Lazarett «einige der furchtbarsten Fälle von Verstümmelung begegnet» [7] seien, was sich unmittelbar in Antikriegsgedichten niedergeschlagen haben soll. Man kann das getrost ins Reich der Erfindungen verweisen: Dass sich im Lazarett auch Schwerstverletzte befanden, ist zwar durchaus möglich, nur hatte der Dichter keinen Kontakt zu ihnen, denn er versah seinen Dienst vom 1. Oktober 1918 bis zu seinem Ausscheiden aus der Armee am 9. Januar 1919 auf der Station D, auf der sich neben einigen Ruhrkranken ausschliesslich Geschlechtskranke befanden. Literaturgeschichtlich vollkommen ohne Belang war die Zeit, die Brecht dort verbrachte, aber dennoch nicht. Er nutzte sie, um ein «Lied an die Kavaliere der Station $\mathrm{D}$ » zu verfassen, von dem nur die erste Strophe erhalten ist:

O wie brannte euch der Liebe Flammen

Als ihr jung und voller Feuer ward

Ach der Mensch haut halt das Mensch zusammen

Das ist nun einmal so seine Art [15]

$\mathrm{Ob}$ auch die «Legende vom toten Soldaten», die später als «Ballade vom toten Soldaten» in den 4. Akt von «Trommeln in der Nacht» einging, während der Zeit im Lazarett oder schon früher entstand, ist hingegen unklar [16].

Die Wirkung dieser Ballade war nicht gerade gering: Bei den Nationalsozialisten soll sie Brecht einen Platz auf einer Liste der im Falle der Machtübernahme sofort zu verhaftenden Personen eingebracht haben, und zwar auf Platz fünf [2]. Kurt Tucholsky äusserte sich später anerkennend: «Den Preußen hats ja mancher besorgt so gegeben hats ihnen noch keiner ...» [17].

Was das Studium betrifft, so hat Brecht bereits am für ehemalige Soldaten obligatorischen Zwischensemester von Februar bis Ende März 1920 nicht mehr teilgenommen. Im Februar 1920 teilte er einer Freundin mit, das Studium aufgeben zu wollen, um fortan Stücke zu schreiben [14] - eine, wie wir heute wissen, offensichtlich richtige Entscheidung.

Das eingangs erwähnte Zitat schliesslich ist einfach eine Fehlübersetzung aus dem Englischen:

If the doctor ordered me: «Amputate a leg, Brecht!», I would answer: "Yes, your excellency", and cut off the leg. If I was told: «make a trepanning", I opened the man's skull and tinkered with his brains ... [18]

Offensichtlich hatte jemand Indikativ und Konjunktiv verwechselt. 